a point which lies in the polar $r-2$ flat of $\alpha$ qua $f(\alpha)=0$. Whence it follows that any $r-2$ flat $\Sigma u_{i} \alpha_{i}=0$ is invariant to each of the infinitesimal transformations of $\Gamma$ represented by the poles of this flat qua $f(\alpha)=0$; and thus, if the poles of this flat do not all lie in an $r-2$ flat, it follows that the infinitesimal transformations of $G$ represented by points in the $r-2$ flat $\Sigma u_{i} \alpha_{i}=0$ generate an invariant subgroup of $G$.

If the adjoint group $\Gamma$ has a quadratic invariant of non-zero discriminant, for a proper choice of the $X$ 's, we shall have $c_{i j k}+c_{i k j}=0(i, j, k=1,2, \cdots, r)$. In this case the condition that $f(\alpha)$ shall be invariant to $\Gamma$ is that

$$
\Sigma \alpha_{i} E_{i}\left(\frac{\partial f(\alpha)}{\partial \alpha_{1}}, \cdots, \frac{\partial f(\alpha)}{\partial \alpha_{r}}\right)=0,
$$

where $E_{i}$ is the matrix whose constituent in the $\mu$ th row and $\nu$ th column is $c_{i_{\nu}}(i, \mu, \nu=1,2, \cdots, r)$; or, what is the same thing, is that

$$
\left(\Sigma \alpha_{i} X_{i}, \Sigma \frac{\partial f(\alpha)}{\partial \alpha_{i}} X_{i}\right)=0
$$

for all values of the $\alpha$ 's. Whence it follows, if $f(\alpha)$ is a second invariant of $\Gamma$, that the infinitesimal transformation $\Sigma u_{i} X_{i}$ is commutative with every infinitesimal transformation of $G$ represented by a pole, qua $f(\alpha)=0$, of the $r-2$ flat $\Sigma u_{i} \alpha_{i}=0$; and, if these poles do not all lie in any $r-2$ flat, it follows that $\Sigma u_{i} X_{i}$ is an exceptional infinitesimal transformation.

F. N. Cole, Secretary.

\title{
THE EQUATION OF A RATIONAL PLANE CURVE DERIVED FROM ITS PARAMETRIC EQUATIONS (SECOND PAPER).
}

BY PROFESSOR J. E. ROWE.

(Read before the American Mathematical Society, February 24, 1917.)

As this is the second article on the same subject published by the author in the Bulletin, it is desirable to inform the reader at once that the method of deriving the equation of a rational plane curve from its parametric equations described in this paper is published not merely because it is a new 
method but also because in many respects it is an improvement upon previous methods. To verify this statement the reader is referred to the processes given by Salmon (Higher Plane Curves, \$ 44), by Grace and Young (Algebra of Invariants, § 254), and to the method of my former article,* which was also developed independently by Mr. H. W. Richmond, of Cambridge University, but not published by him until after my article had appeared.

As in the preceding article, it seems best for the sake of readability to present the method through the instrumentality of the easiest illustrations.

First, let

$$
\rho x_{i}=a_{i} t^{2}+b_{i} t+c_{i} \quad(i=0,1,2)
$$

be the parametric equations of a conic or $R^{2}$. By multiplying $\dagger$ each of these equations by $k$, transposing all terms to the same side, and replacing $-k \rho x_{i}$ by $\sigma x_{i}$, we obtain

$$
a_{i} k t^{2}+b_{i} k t+c_{i} k+\sigma x_{i}=0 \quad(i=0,1,2) .
$$

Solving (2) for the ratios of $k t^{2}, k t, k, \sigma$ regarded as independent quantities, we have

(3) $k t^{2}:-k t: k:-\sigma=|b c x| \ddagger:|a c x|:|a b x|:|a b c|$.

From (3)

$$
-t=\frac{k t^{2}}{-k t}=\frac{-k t}{k}=\frac{|b c x|}{|a c x|}=\frac{|a c x|}{|a b x|} .
$$

Hence from (4)

$$
\begin{aligned}
& |a b x| t+|a c x|=0 \\
& |a c x| t+|b c x|=0
\end{aligned}
$$

Eliminating $t$ we have

$$
\left|\begin{array}{ll}
|a b x| & |a c x| \\
|a c x| & |b c x|
\end{array}\right|=0
$$

and this is the equation of the $R^{2}$ of (1). Obviously in this

* This Bulletin, vol. 22, No. 7 (April, 1916), pp. 338-340. Also, see Professor Richmond's article, vol. 23, No. 2 (November, 1916), pp. 90-91. $\dagger$ This is merely a matter of personal preference.

$\ddagger \mathrm{By}|a b x|$ is meant the three-rowed determinant $\left|\begin{array}{lll}a_{0} & b_{0} & x_{0} \\ a_{1} & b_{1} & x_{1} \\ a_{2} & b_{2} & x_{2}\end{array}\right|$. 
simple case the same result might have been obtained by simplifying the last equality in (4).

Further, let

$$
\rho x_{i}=a_{i} t^{3}+b_{i} t^{2}+c_{i} t+d_{i} \quad(i=0,1,2)
$$

be the parametric equations of the rational plane cubic $R^{3}$. Following (2), these may be written

$$
a_{i} k t^{3}+b_{i} k t^{2}+c_{i} k t+d_{i} k+\sigma x_{i}=0 \quad(i=0,1,2) .
$$

These may be arranged in a form similar to (2) in a number of ways; for example,

$$
a_{i} t \cdot k t^{2}+b_{i} t \cdot k t+\left(c_{i} t+d_{i}\right) k+\sigma x_{i}=0,
$$

$$
\left(a_{i} t+b_{i}\right) k t^{2}+c_{i} \cdot k t+d_{i} k+\sigma x_{i}=0 .
$$

Solving these equations for the ratios, we find

(10) $k t^{2}:-k t: k:-\sigma$

$$
=(|b c x| t+|b d x|):(|a c x| t+|a d x|):|a b x| t: U_{3}{ }^{*}
$$

$(11)=|c d x|:(|a d x| t+|b d x|):(|a c x| t+|b c x|): U_{3}^{\prime}$.

As in (4), it follows from (10) that

$$
\begin{aligned}
-t & =(|b c x| t+|b d x|) /(|a c x| t+|a d x|) \\
& =(|a c x| t+|a d x|) /|a b x| t ;
\end{aligned}
$$

similarly, from (11)

$$
\begin{aligned}
-t & =|c d x| /(|a d x| t+|b d x|) \\
& =(|a d x| t+|b d x|) /(|a c x| t+|b c x|) .
\end{aligned}
$$

Multiplying and transposing in (12) and (13) yields the system of equations

$$
\begin{aligned}
& |a b x| t^{2}+|a c x| t+|a d x|=0, \\
& |a c x| t^{2}+(|a d x|+|b c x|) t+|b d x|=0, \\
& |a d x| t^{2}+|b d x| t+|c d x|=0 .
\end{aligned}
$$

The determinant of (14) equated to zero is the equation of the $R^{3}$.

${ }^{*} U_{3}$ and $U_{3}{ }^{\prime}$ need not be calculated, as they are not used in subsequent 
In deriving the equation of the $R^{n}$ whose parametric equations are

$$
a_{i} k t^{n}+b_{i} k t^{n-1}+\cdots+\sigma x_{i}=0 \quad(i=0,1,2),
$$

it is never necessary to solve for the ratios $k t^{2}, k t, k, \sigma$ in more than $(n+1) / 2$ ways. The third equation of (14) might have been obtained from the first by interchanging $a_{i}$ and $d_{i}, b_{i}$ and $c_{i}$ with the subsequent change of $t$ to $1 / t$. Obviously the second equation of (14) is invariant under this transformation. Further, if we make $n=5$ in (15), the equation of the $R^{5}$ whose parametric equations are so obtained may be found by eliminating the powers of $t$ from five equations. Three of these five equations

$$
\begin{aligned}
& |a b x| t^{4}+|a c x| t^{3}+|a d x| t^{2}+|a e x| t+|a f x|=0, \\
& |a c x| t^{4}+(|a d x|+|b c x|) t^{3}+(|a e x|+|b d x|) t^{2} \\
& +(|a f x|+|b e x|) t+|b f x|=0, \\
& |a d x| t^{4}+(|a e x|+|b d x|) t^{3} \\
& +(|a f x|+|b e x|+|c d x|) t^{2}+(|b f x|+|c e x|) t \\
& +|c f x|=0 .
\end{aligned}
$$

may be obtained by solving for the ratios in the two ways indicated by

$$
\begin{aligned}
& a_{i} t^{3} \cdot k t^{2}+b_{i} t^{3} \cdot k t+\left(c_{i} t^{3}+d_{i} t^{2}+e_{i} t+f_{i}\right) k+\sigma x_{i}=0, \\
& \left(a_{i} t^{3}+b_{i} t^{2}\right) k t^{2}+c_{i} t^{2} \cdot k t+\left(d_{i} t^{2}+e_{i} t+f_{i}\right) k+\sigma x_{i}=0 .
\end{aligned}
$$

Using the transformation mentioned above (which in this case consists in interchanging $a_{i}$ and $f_{i}, b_{i}$ and $e_{i}, c_{i}$ and $d_{i}$ with the subsequent change of $t$ to $1 / t)$, the two remaining equations of the five may be obtained from the first two of (16). The third equation of (16) is invariant under the transformation. Obviously equations (15) can be solved for the ratios in as many ways as the coefficients of $k t^{2}, k t$, and $k$ can be selected. It will be found convenient in actual work to use the scheme partly indicated in equations (17). That is, in the first and second solutions of equations (15) the coefficients of $k t^{2}$ and $k t$ are $a_{i} t^{n-2}, b_{i} t^{n-2}$ and $a_{i} t^{n-2}+b_{i} t^{n-3}, c_{i} t^{n-3}$ respectively; according to the same scheme in the third solution the coefficients of $k t^{2}$ and $k t$ are $a_{i} t^{n-2}+b_{i} t^{n-3}+c_{i} t^{n-4}$, $d_{i} t^{n-4}$, etc. 
In (5) we have the equations of two lines different for different values of $t$, and the locus of their intersection is the $R^{2}$. In (14) we have three concurrent variable lines, and the locus of their intersection is the $R^{3}$. Hence in general the method consists in finding the locus of the points of concurrence of $n$ concurrent lines subject to the condition that this point of concurrence be on the $R^{n}$.

Pennsylvania State College, February, 1917.

\section{FORD'S STUDIES ON DIVERGENT SERIES AND SUMMABILITY.}

Studies on Divergent Series and Summability. By Walter Burton Ford. Michigan Science Series, Volume II. New York, The Macmillan Company, 1916. xi +194 pp.

DURING the past twenty years there has been an ever increasing interest in the study of divergent series and their applications. Naturally a coexistent phenomenon has been a very large expansion in the volume of literature on this subject. An idea of the amount of this expansion may be gathered from the bibliography of Professor Ford's book, which, while not exhaustive, contains a list of some two hundred books and memoirs (principally memoirs), of which all but about twenty have appeared from 1895 on.

Thus it has become more and more difficult for one who has not followed recent work on divergent series to ascertain readily the known results in a certain branch of that field or the methods that have proved fruitful in studying certain aspects of the subject. This is alike a handicap for the experienced research worker whose investigations in other fields have naturally led to a consideration of divergent series, and to the beginner in research who feels attracted toward the subject of divergent series and wishes to orient himself rapidly in the field in order to find the avenues that may lead to new results.

To both of these classes of readers, as well as to many others, Professor Ford's admirable work will undoubtedly prove a boon. It presents in clear and concise fashion the fundamental features of each of the two grand divisions of divergent series, namely, asymptotic series and summable series, and in 